\title{
Clinical analysis of 198 cases of western medicine adverse reaction
}

\author{
Yanhong Chen* \\ Inner Mongolia Baogang Hospital, Inner Mongolia, China
}

Received: January 22, 2018

DOI: $10.14725 /$ dcc.v5n1p30
Accepted: March 1, $2018 \quad$ Online Published: March 10, 2018

URL: http://dx.doi.org/10.14725/dcc.v5n1p30

\begin{abstract}
Objective: To discuss the clinical symptoms of western medicine adverse reaction, and to provide basis for clinical rational drug use.

Methods: Clinical data of 198 patients with adverse drug reactions were retrospectively analyzed in our hospital. Adverse reactions clinical manifestations, the types of drugs and the route of medication were summarized.

Results: The first two common adverse reactions were anti-infective drugs and cardiovascular system drugs respectively. Intramuscular injection and intravenous administration were two main routes of medication for adverse drug reactions. The main clinical symptoms of adverse reactions were digestive tract and anaphylactic shock etc. After treatment, 184 patients recovered and 14 patients got better.

Conclusions: In order to reduce the incidence of adverse reactions as far as possible, clinicians should do a good job of rational use of drugs and attach importance to the safety of drug use. Adverse reactions monitoring can reduce the occurrence of adverse reactions and ensure the safety of clinical treatment.
\end{abstract}

Key Words: Western medicine adverse reaction, Anti-infection, Respiratory system

Studies have shown that all the drugs can produce varying degrees of adverse reactions to the human body. These adverse reactions may cause disability, malformation and so forth. The clinical data of 198 patients with adverse reactions on western medicine in our hospital were retrospectively analyzed and the clinical symptoms of adverse reactions were discussed in order to provide scientific basis for clinical use of drugs.

\section{Information and methods}

\subsection{General information}

198 patients with adverse reactions on western medicine from January 2013 to August 2013 were treated as objects, including 103 males and 95 females, ranging from 23 to 63 years old, with an average of $(36.2 \pm 4.5)$ years old. The basic data of all patients were summarized and analyzed in detail.

\subsection{Methods}

The clinical data of 198 patients were analyzed retrospectively, and the classification of western medicine, clinical manifestations and ways of administration were summarized and analyzed.

\subsection{Unreasonable drug use standard}

According to the related standards of irrational drug use of Prescription Management Methods and Interim Regulations

*Correspondence: Yanhong Chen; E-mail: dcc69@ncspress.com; Address: Inner Mongolia Baogang Hospital, Inner Mongolia, China. 
on the Administration of Pharmacy in Medical Institutions etc. issued by National Ministry of Health and Administration of Western Medicine, the cases of adverse reactions of western medicine were analyzed. ${ }^{[1]}$

\section{Results}

\subsection{Adverse reaction drug types}

There were 4 kinds of drugs involved in adverse reaction. The number of variety was 58 . The adverse reaction occurred 198 times. The first two common adverse reaction drugs were anti-infective drugs (66 cases, proportion $33.33 \%$ ), the cardiovascular system drugs ( 58 cases, proportion $29.29 \%$ ), respiratory system drugs (42 cases, proportion $21.21 \%$ ) and digestive system drugs (22 cases, proportion $11.11 \%)$.

\subsection{Clinical manifestation}

In 198 patients with adverse reactions, there were 87 cases of digestive system symptoms (diarrhea, vomiting, nausea, etc.), accounting for $43.94 \%$; 47 cases of nervous system symptoms, accounting for $23.74 \%$; 43 cases of anaphylactic shock, accounting for $21.72 \% ; 21$ cases of cardiovascular diseases, accounting for $10.61 \%$.

\subsection{Route of medication}

There were 4 different routes of medication for 198 patients namely intramuscular injection, intravenous injection, oral medication and topical medication, which accounted for $47.98 \%$ (95/198), $23.74 \%$ (47/198), 19.70\% (39/198), $8.59 \%(17 / 198)$ respectively. The most common ways to produce adverse reactions to drugs were muscle injection and intravenous injection.

\subsection{Treatment effect}

In 198 patients with adverse drug reactions, 184 recovered and 14 improved in varying degrees.

\section{Discussion}

To ensure the high drug using safety of the patients and reduce the incidence of adverse reactions as far as possible, doctors should fully understand the adverse drug reactions in order to improve the level of clinical drug use. At the same time, the monitoring of adverse reactions for drug is also an effective method. In addition, doctors should comply with the requirements of rational drug use in daily work fully to promote rational clinical use. In this study, two major adverse reactions drug were anti-infective drugs and cardiovascular system drugs; intramuscular injection and intravenous administration were two major routes for medication of adverse reactions; and the clinical symptoms of adverse reactions were mainly digestive tract and anaphylactic shock and so on. After treatment, 184 patients recovered and 14 patients improved. Therefore, in order to reduce the incidence of adverse reactions as much as possible, the clinicians should make good use of drugs reasonably and attach importance to the safety of drug use, and monitor the status of adverse reactions to reduce the occurrence of adverse reactions and ensure the safety of clinical treatment.

\section{Conflicts of Interest Disclosure}

The authors have no conflicts of interest related to this article.

[1] Li YH, Wang CX. Clinical Analysis 45 Cases with Western Medicine Adverse Reactions. Chinese Medicine Guide. 2014; 9: 132-133. 